\title{
MODELAGEM MATEMÁTICA DA EXTRAÇÃO DO ÓLEO DE MACAÍBA (Acrocomia acuelata) COM DIÓXIDO DE CARBONO SUPERCRÍTICO
}

\author{
A. D. PEÇANHA ${ }^{1}$, L. STRAGEVITCH ${ }^{1}$, L.A.L. SOARES ${ }^{2}$ e L. DANIELSKI ${ }^{1}$ \\ ${ }^{1}$ Universidade Federal de Pernambuco, Departamento de Engenharia Química, \\ Laboratório de Combustíveis (LAC). \\ ${ }^{2}$ Universidade Federal de Pernambuco, Departamento de Ciências Farmacêuticas, \\ Laboratório de Farmacognosia. \\ E-mail para contato: andreadacalpeanha@yahoo.com.br
}

\begin{abstract}
RESUMO - O Brasil abriga uma das maiores biodiversidades do mundo por apresentar diferentes tipos de biomas e condições climáticas favoráveis. Registra-se nas últimas décadas um aumento pelo interesse em recuperar bioativos oriundos de fontes naturais, principalmente para o desenvolvimento de alimentos funcionais e nutracêuticos. Porém, os processos tradicionais envolvem etapas onde se faz necessário o uso de altas temperaturas e solventes orgânicos. Neste contexto, a extração supercrítica (ESC) com dióxido de carbono se mostra eficaz devido às condições operacionais brandas e facilidade de separação soluto/solvente. No presente estudo, aplicou-se a ESC para a obtenção do óleo da polpa de macaíba; a técnica foi conduzida com pressões variando de 100 a 200 bar e temperaturas de 40 e $55^{\circ} \mathrm{C}$. Os dados experimentais foram modelados utilizando os modelos propostos por ESQUÍVEL et al.(1999) e MARTÍNEZ et al. (2003).
\end{abstract}

\section{INTRODUÇÃO}

Desde os primórdios da humanidade, o uso de plantas aromáticas e medicinais está ligado ao avanço das civilizações, a princípio utilizadas exclusivamente para fins alimentares e, em seguida para o tratamento e prevenção de doenças (BIAZZI, 2004). Atualmente, o interesse pela recuperação de bioativos de fontes naturais tem aumentado principalmente para o desenvolvimento de alimentos funcionais e nutracêuticos.

Neste contexto, a extração supercrítica (ESC) vem se tornando uma tecnologia promissora, uma vez que proporciona maior seletividade, não faz uso de solventes tóxicos e ainda apresenta um tempo de operação reduzido, quando comparada a outras técnicas de extração (SERRA et al., 2010). Outro fator preponderante na escolha da referida técnica, diz respeito às limitações legais para o uso de diversos solventes orgânicos em processos extrativos, direcionando assim os estudos para aqueles que empregam solventes do tipo GRAS “Generally Recognized As Safe”.

De acordo com BRUNNER (1994), o uso da ESC justifica-se pelo emprego de temperaturas mais amenas durante o processo, tornando a técnica mais adequada para compostos termolábeis e 
ainda pela eficiência da separação do extrato/solvente, a qual é dada apenas pela redução da pressão ao fim da extração. Portanto, conhecer as condições cinéticas e termodinâmicas envolvidas na utilização de fluidos supercríticos empregados na extração de substâncias contidas em vegetais permite o aprimoramento e otimização da técnica.

A fim de buscar a otimização do processo, a avaliação das curvas de extração se faz necessária. As curvas de extração são geralmente dadas em função da vazão do solvente e da concentração do soluto na fase solvente, sendo determinada pela massa total de óleo extraído em função do tempo ou massa do solvente utilizada na técnica (BRUNNER, 1994).

Uma gama de pesquisadores propuseram modelos matemáticos para a descrição das curvas de extração. Dentre os modelos disponíveis na literatura encontram-se o modelo de SOVOVÁ (1994) e o modelo logístico de MARTíNEZ et al. (2003), os quais se baseiam na integração diferencial do balanço de massa no interior do extrator. Outros pesquisadores propuseram modelos análogos à segunda lei de Fick para transferência de calor, como os modelos de CRANK (1975) e o de GASPAR et al. (2003). Adicionalmente, modelos empíricos como o apresentado por ESQUÍVEL et al. (1999) também podem ser encontrados na literatura.

Neste estudo aplicou-se a ESC na obtenção do óleo da polpa da macaíba (Acrocomia acuelata com pressões entre 100 e 200 bar e temperaturas de operação de 40 e $55^{\circ} \mathrm{C}$. Os dados experimentais foram modelados matematicamente utilizando os modelos propostos por ESQUÍVEL at al. (1999) e MARTÍNEZ et al. (2003). Os erros médios quadráticos obtidos foram considerados satisfatórios.

\section{MATERIAL E MÉTODOS}

\subsection{Metodologia Experimental}

Os frutos de macaíba (Figura 1) foram adquiridos em um mercado local, situado na cidade do Recife (PE). A polpa dos frutos foi submetida à secagem em estufa com circulação forçada de ar, temperatura de $45{ }^{\circ} \mathrm{C} \pm 2$ por aproximadamente $24 \mathrm{~h}$. Após a secagem, as polpas foram acondicionadas em recipiente plástico, armazenadas em freezer comercial (temperatura aproximada de $-18^{\circ} \mathrm{C}$ ) e mantidas assim até o momento dos ensaios.

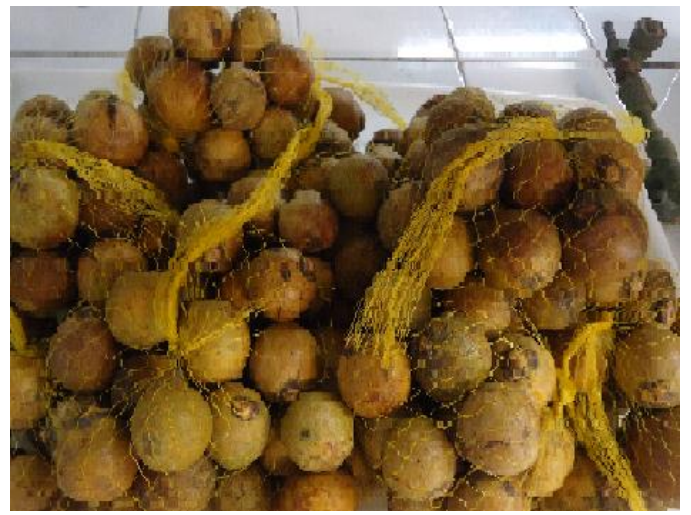


Figura 1 - Frutos de macaíba (Acrocomia acuelata).

Para os experimentos, utilizou-se um equipamento de ESC (Figura 2), acoplado a um cilindro de dióxido de carbono $\left(\mathrm{CO}_{2}\right)$ com tubo pescador e os extratos obtidos foram recolhidos em frascos de vidro âmbar, previamente tarados. Compactou-se aproximadamente $40 \mathrm{~g}$ da polpa seca e moída, dentro do extrator, formando-se assim o leito fixo.

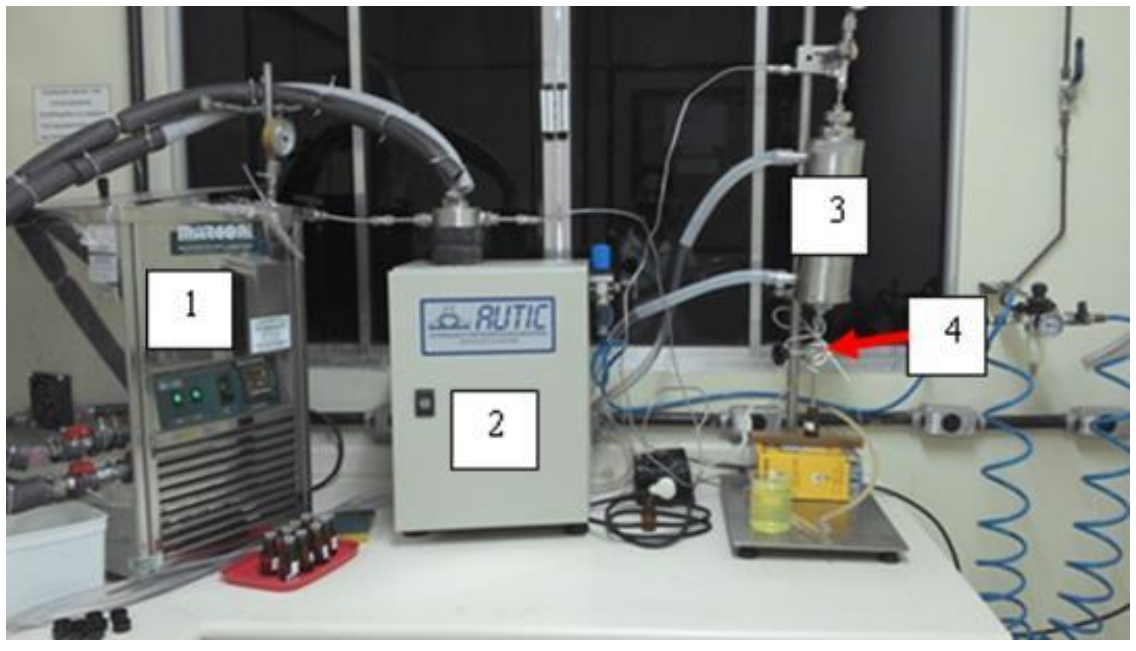

Figura 2 - Equipamento de extração supercrítica: (1) Banho termostático; (2) Bomba de $\mathrm{CO}_{2}$; (3) Extrator; (4) Válvula micrométrica.

\subsection{Modelagem Matemática}

Os dados experimentais obtidos foram correlacionados usando os modelos propostos por ESQUÍVEL et al. (1999) e MARTÍNEZ et al. (2003).

O modelo logístico de MARTíNEZ et al. (2003) baseia-se no balanço diferencial de massa no leito do extrator, considerando o balanço tanto na fase sólida quanto na fase fluida, desprezando o acúmulo e a dispersão na fase fluida. As curvas de extração global são representadas pela Eq.1.

$$
m_{e x t}=\frac{m_{t}}{e\left(b t_{m}\right)}\left\{\frac{1+e\left(b t_{m}\right)}{1+e\left[b\left(t_{m}-t\right)\right]}-1\right\}
$$

sendo $m_{\text {ext }}$ a massa de extrato obtida; $m_{t}$ a massa inicial total de sólido no leito fixo de partículas; $t$ o tempo de extração e $b$ e $t_{m}$ os parâmetros do modelo.

ESQUÍVEL et al. (1999) propuseram um modelo análogo à cinética de crescimento microbiano de Monod para descrever o comportamento das curvas de extração. O modelo empírico é representado por meio da Eq. 2. 


$$
m_{e x t}(t)=X_{0} F\left(\frac{t}{b_{e}+t}\right)
$$

sendo $m_{\text {ext }}(t)$ a massa extraída em função do tempo; $t$ o tempo de extração; $F$ a massa do material; $X_{O}$ a razão entre a massa inicial do soluto e a fase sólida e $b_{e}$ o parâmetro ajustável do próprio modelo.

\section{RESULTADOS E DISCUSSÃO}

Os ensaios foram realizados nos níveis de temperatura e pressão apresentados na Tabela 1. De acordo com experimentos prévios realizados com extrator de Soxhlet, a polpa de macaíba apresentou composição média de $26 \%$ de óleo em massa. Para os experimentos listados, a média das vazões de $\mathrm{CO}_{2}$ foi de $1,55 \mathrm{~g} / \mathrm{min}$.

Tabela 1 - Condições operacionais aplicadas na ESC de óleo de polpa de macaíba.

\begin{tabular}{|c|c|c|c|}
\hline $\mathbf{T}\left({ }^{\circ} \mathbf{C}\right)$ & $\mathbf{P}(\mathbf{b a r})$ & Densidade do $\mathbf{C O}_{\mathbf{2}}{ }^{(\mathbf{a})}\left(\mathbf{k g} / \mathbf{m}^{\mathbf{3}}\right)$ & Rendimento (massa, \%) \\
\hline 40 & 100 & 629,96 & 10,23 \\
& 200 & 841,31 & 29,16 \\
\hline 55 & 150 & 658,70 & 17,16 \\
& 200 & 756,17 & 24,25 \\
& \multicolumn{2}{|c}{} \\
\hline
\end{tabular}

A partir da análise dos dados apresentados na Tabela 1 percebeu-se que os maiores rendimentos de extração foram obtidos quando a densidade do $\mathrm{CO}_{2}$ atingiu valores mais altos (maior pressão em temperatura constante), apresentando um maior efeito na solubilização do soluto. $\mathrm{O}$ aumento da temperatura diminuiu a densidade do solvente a pressão constante, o que foi responsável pelo menor rendimento para o experimento conduzido a $55^{\circ} \mathrm{C}$ e 200 bar em comparação ao conduzido a $40{ }^{\circ} \mathrm{C}$ e 200 bar.

A partir dos dados experimentais para o sistema óleo de macaíba $+\mathrm{CO}_{2}$ foi possível modelar as curvas de extração com o auxílio do software Mass Transfer (CORREIA et al., 2006). A Figura 3 apresenta os resultados obtidos a partir do ensaio conduzido na temperatura de $40{ }^{\circ} \mathrm{C}$ e pressão de 100 bar. 


\section{9 a 22 de outubro de 2014 \\ Florianópolis/SC}

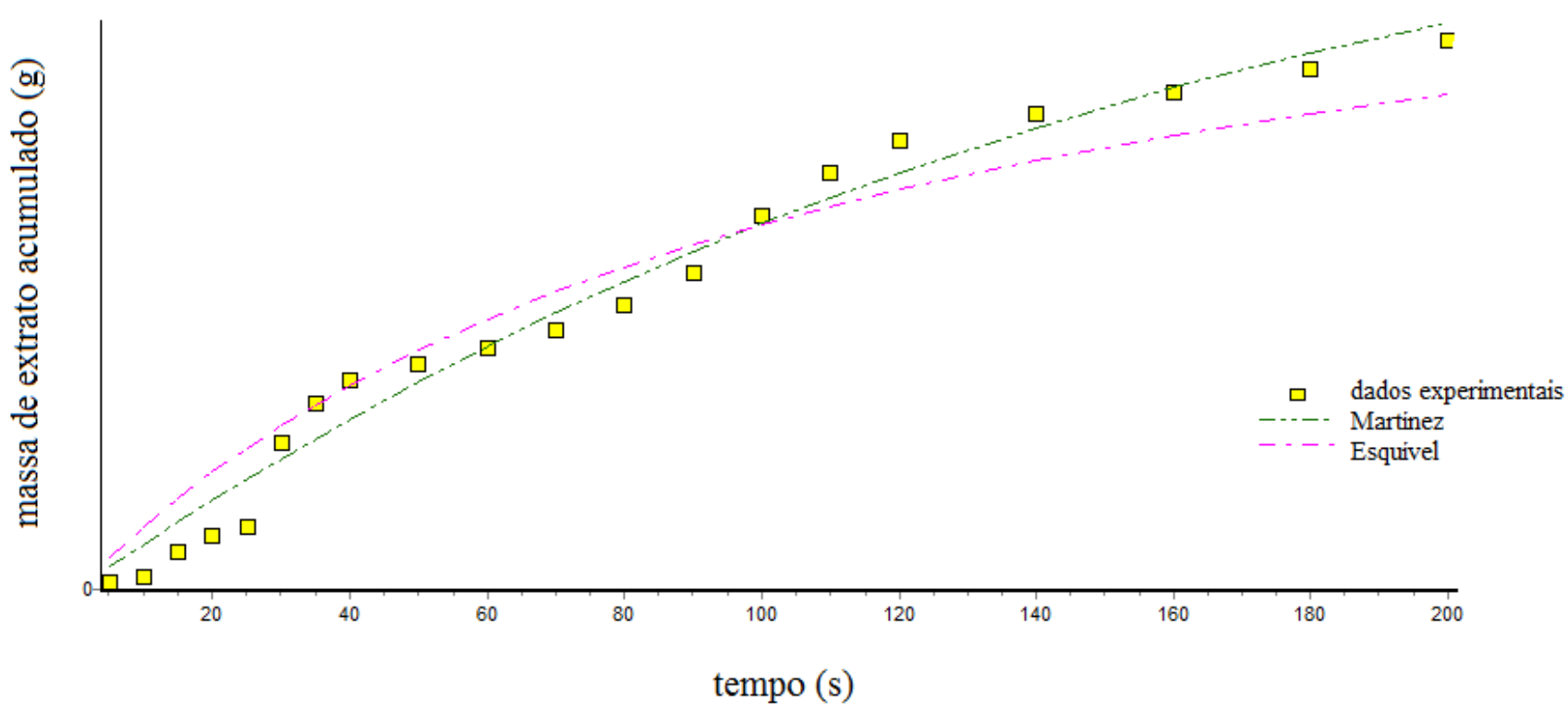

Figura 3 - Resultados obtidos na modelagem da ESC do óleo de polpa de macaíba a $40{ }^{\circ} \mathrm{C}$ e 100 bar.

Nas Figuras 4, 5 e 6 são apresentados os resultados obtidos nas condições experimentais de $40^{\circ} \mathrm{C}$ e 200 bar; $55^{\circ} \mathrm{C}$ e 150 bar; $55^{\circ} \mathrm{C}$ e 200 bar, respectivamente.

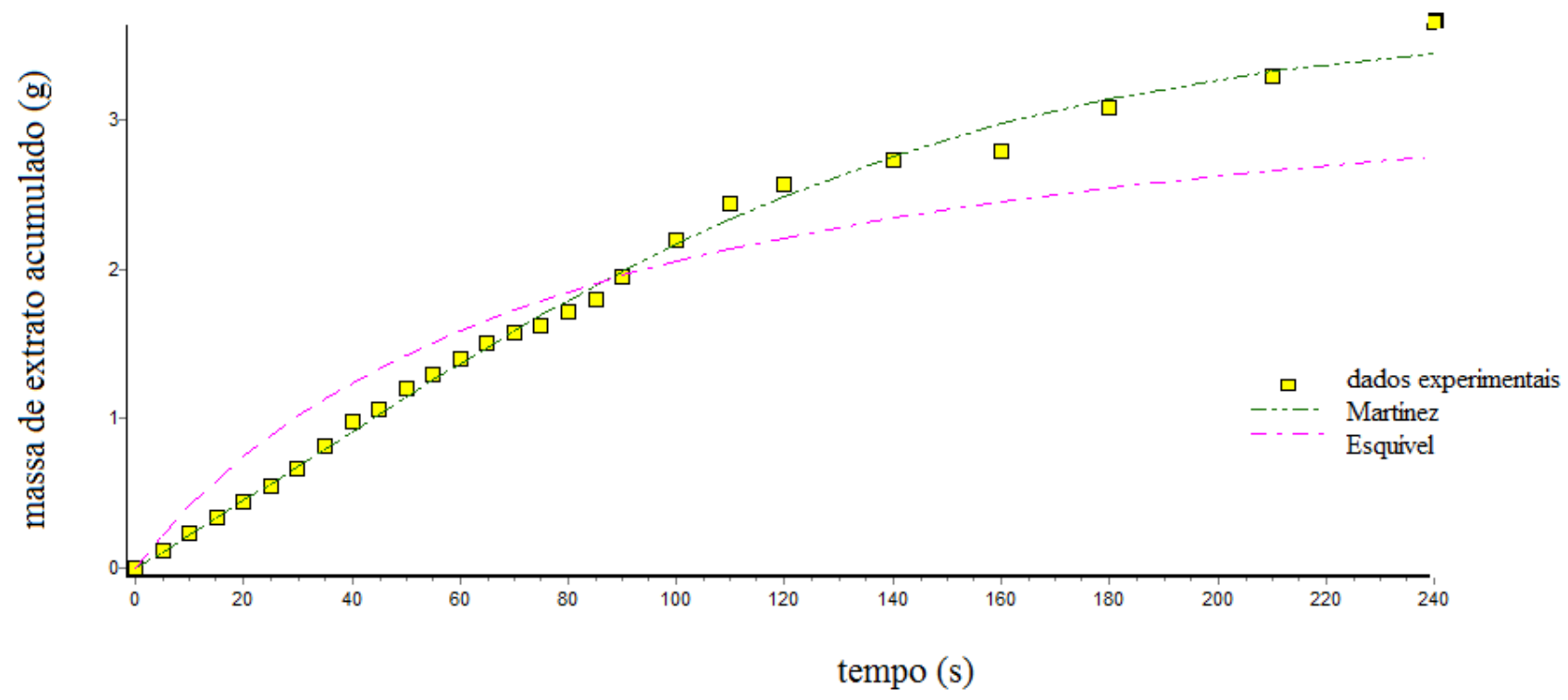

Figura 4 - Resultados obtidos na modelagem da ESC do óleo de polpa de macaíba a $40{ }^{\circ} \mathrm{C}$ e 200 bar. 


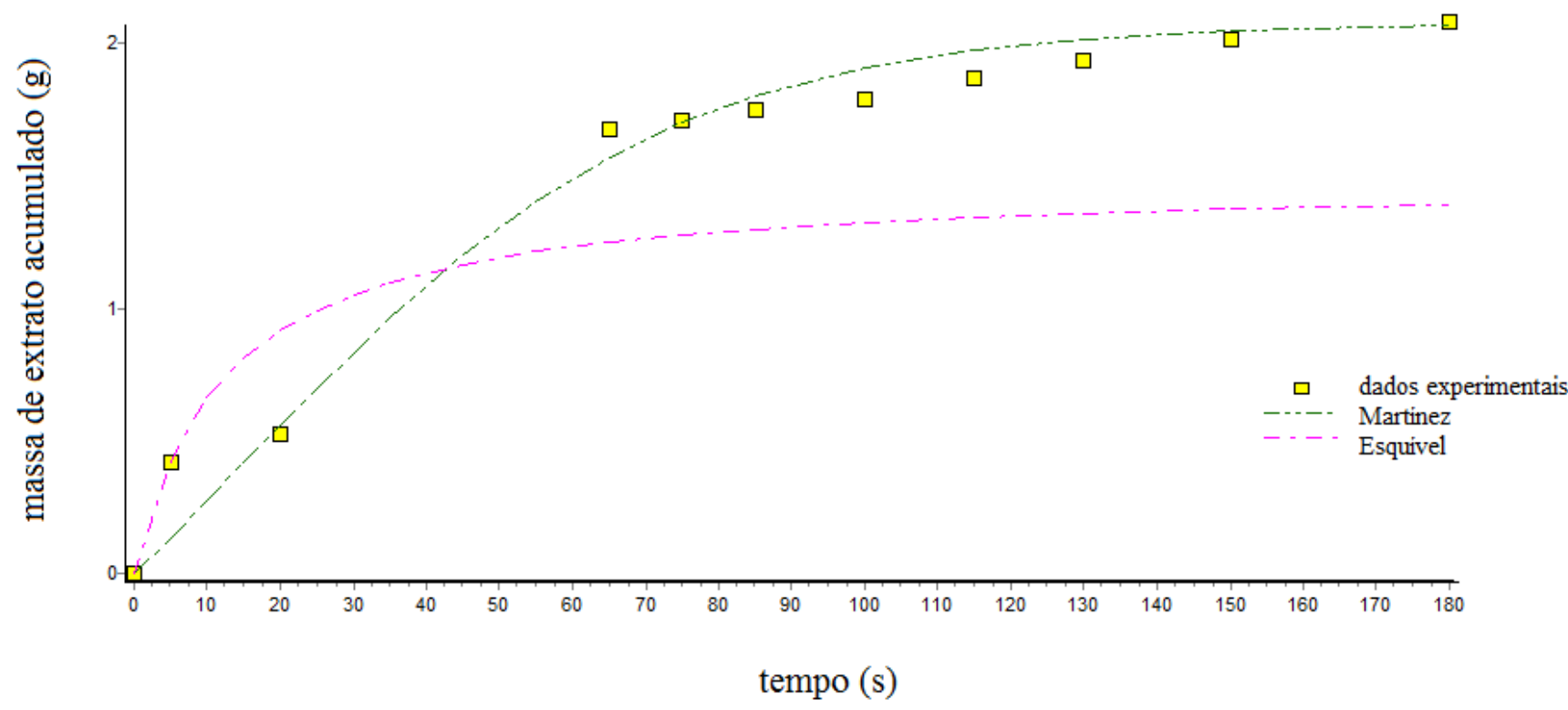

Figura 5 - Resultados obtidos na modelagem da ESC do óleo de polpa de macaíba a $55^{\circ} \mathrm{C}$ e 150 bar.

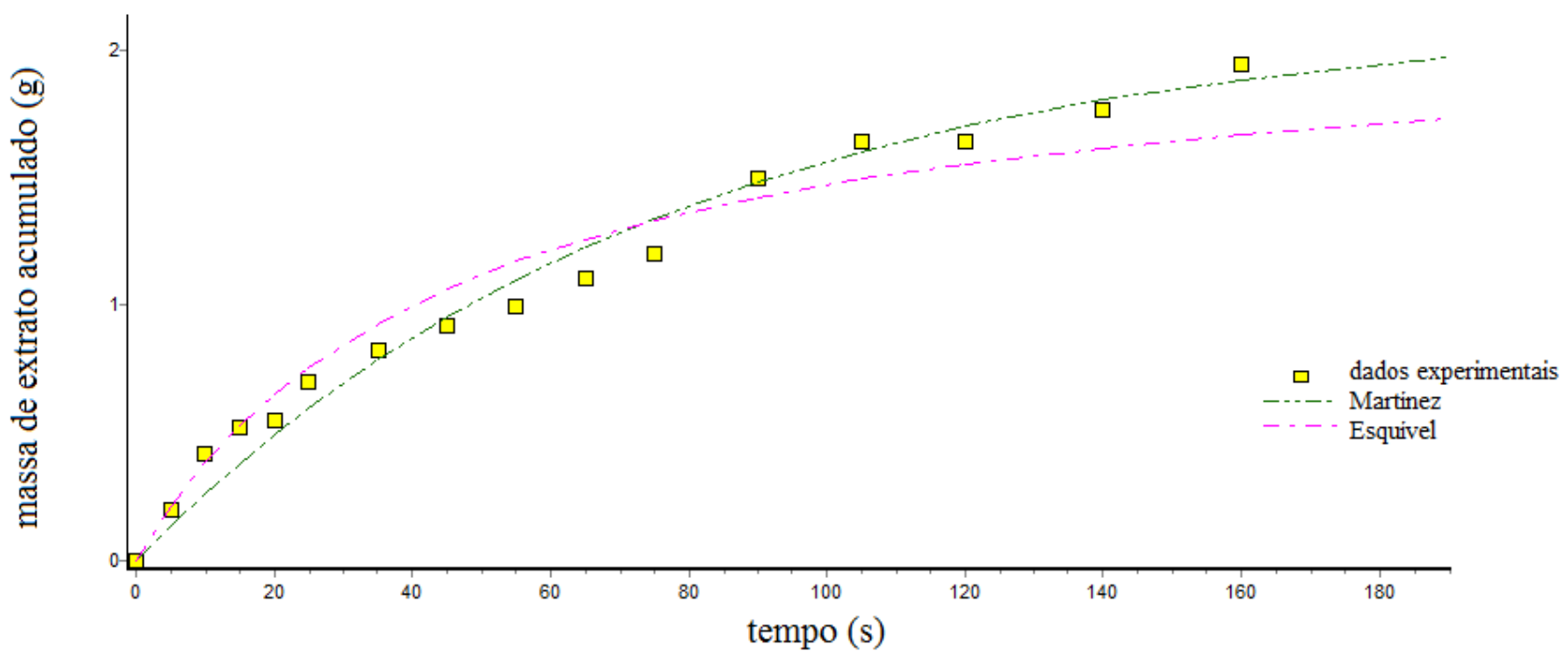

Figura 6 - Resultados obtidos na modelagem da ESC do óleo de polpa de macaíba a $55^{\circ} \mathrm{C}$ e 200 bar.

Os erros médios quadráticos obtidos a partir da modelagem matemática para o sistema óleo de macaíba $+\mathrm{CO}_{2}$ estão apresentados na Tabela 2. Quando considerando as temperaturas constantes, os maiores erros foram obtidos nas pressões mais elevadas. Porém, observou-se também que os maiores 
erros médios quadráticos foram obtidos na temperatura de $55{ }^{\circ} \mathrm{C}$ para os dois níveis de pressão avaliados (150 e 200 bar). Sabe-se que, com o aumento da temperatura, o aumento da pressão de vapor do soluto pode influenciar significativamente a sua solubilidade no solvente.

Tabela 2 - Erros médios quadráticos obtidos na ESC do óleo de polpa de macaíba para os dois modelos investigados.

\begin{tabular}{|c|c|c|c|}
\hline $\mathbf{T}\left({ }^{\circ} \mathbf{C}\right)$ & $\mathbf{P}(\mathbf{b a r})$ & Esquível et al. & Martínez et al. \\
\hline 40 & 100 & 0,0030 & 0,0044 \\
& 200 & 0,1078 & 0,0049 \\
\hline 55 & 150 & 0,1966 & 0,0300 \\
& 200 & 0,0250 & 0,0090 \\
\hline
\end{tabular}

Finalizando, a Tabela 3 apresenta os valores obtidos para os parâmetros ajustáveis dos modelos utilizados nos experimentos.

Tabela 3 - Parâmetros ajustáveis obtidos na modelagem matemática da ESC de macaíba.

\begin{tabular}{|c|c|c|c|c|}
\hline $\begin{array}{c}\mathbf{T} \\
\left({ }^{\circ} \mathbf{C}\right)\end{array}$ & $\begin{array}{c}\mathbf{P} \\
\text { (bar) }\end{array}$ & $\begin{array}{l}b \times 10^{3} \\
\left(\min ^{-1}\right)\end{array}$ & $\begin{array}{c}\mathbf{t}_{\mathrm{m}} \\
(\mathbf{m i n})\end{array}$ & $\begin{array}{l}\mathbf{b}_{\mathbf{e}} \\
(-)\end{array}$ \\
\hline \multirow[t]{2}{*}{40} & 100 & 0,0011 & 471,659 & 109,7989 \\
\hline & 200 & 0,0158 & 32,0199 & 77,7752 \\
\hline \multirow[t]{2}{*}{55} & 150 & 0,0340 & 14,8399 & 12,4626 \\
\hline & 200 & 0,0132 & $-357,4322$ & 45,7453 \\
\hline
\end{tabular}

\section{Conclusão}

No estudo realizado aplicaram-se dois modelos matemáticos diferentes no ajuste dos dados experimentais da ESC do óleo da polpa de macaíba. Os sistemas operados a $40^{\circ} \mathrm{C} / 100$ bar e a $40^{\circ} \mathrm{C} / 200$ bar foram os que se ajustaram de forma mais satisfatória aos modelos propostos, considerando que a análise foi embasada nos menores valores de erros quadráticos encontrados para os sistemas citados.

\section{Referências}

ANGUS, S.; ARMSTRONG, B.; DE REUCK, K.M. International thermodynamic tables of the fluid 
state: Carbon dioxide. Vol. 3, Pergamon Press, 1976.

BIAZZI, E. O Maravilhoso Poder das Plantas. 18 ed. São Paulo: Casa Publicadora Brasileira, 2004.

BRUNNER, G. Gas Extraction: An Introduction to Fundamentals of Supercritical Fluids and the Application to Separation Processes. New York: Springer, 1994.

CORREIA, J.; MICHIELIN, E. M. Z.; FERREIRA, S. R. S. Estudo de modelos de transferência de massa para processos de extração supercrítica, Florianópolis: 2006, 31 p. Departamento de Engenharia Química e de Alimentos - Universidade Federal de Santa Catarina.

CRANK, J. The Mathematics of Diffusion. Oxford: Clarendon Press, 1975.

ESQUÍVEL, M. M.; BERNARDO-GIL, M. G.; KING, M. B. Mathematical models for supercritical extraction of olive husk oil. J. of Supercrit. Fluids, v. 16, p. 43-58, 1999.

ESQUÍVEL, M. M.; BERNARDO-GIL, M. G.; KING, M. B. Mathematical models for supercritical extraction of olive husk oil. The Journal of Supercritical Fluids, 1999, 16, 43-50.

GASPAR, F.; LU, T.; SANTOS, B.; AL-DURI, B. Modeling the extraction of essential oils with compressed carbon dioxide, Journal. Supercrit. Fluids, 2003, 25, 247-260.

MARTÍNEZ, J.; MONTEIRO, A. R.; ROSA, P. T. V.; MARQUES, M. O. M.; MEIRELES, M. A. A. Multicomponent model to describe extraction of ginger oleoresin with supercritical carbon dioxide. Ind. Eng. Chem. Res., v. 42, p. 1057-1063, 2003.

MARTÍNEZ, J.; MONTEIRO, A.R.; ROSA, P.T.V.; MARQUES, M.O.M.; MEIRELES, M.A.A. Multicomponent model to describe extraction of ginger oleoresin with supercritical carbon dioxide, Ind. Eng. Chem., 2003, 42, 1057-1063.

SERRA, A. et al. Processing Cherries (Prunus avium) Using Supercritical Fluid Technology. Part1: recovery of extract fractions rich in bioactive compounds. Journal of Supercritical Fluids, v. 55, p.184-191, 2010.

SOVOVÁ, H. Rate of the vegetable oil extraction with supercritical CO2. I. Modelling of extraction curves, Chem. Eng. Sci., 1994, 49, 409-415. 